\title{
Maladie chez les voyageurs canadiens et les migrants revenus du Brésil : Données de surveillance de CanTravNet, 2013 à 2016
}

\author{
Boggild $\mathrm{AK}^{1,2^{\star}}$, Geduld J33, Libman $\mathrm{M}^{4}$, Yansouni $\mathrm{CP}^{4}$, McCarthy $\mathrm{AE}^{5}$, Hajek J6, Ghesquiere $\mathrm{W}^{7}$, \\ Vincelette $\mathrm{J}^{8}$, Kuhn $\mathrm{S}^{9}$, Plourde PJ10, Freedman DO ${ }^{11}$, Kain $\mathrm{KC}^{1,12}$
}

\section{Résumé}

Contexte : Compte tenu des Jeux olympiques d'été de 2016, on s'attend à ce que les professionnels de la santé canadiens aient besoin d'information sur les maladies courantes dont pourraient être atteints les voyageurs revenant du Brésil.

Objectif : Déterminer les caractéristiques démographiques et de voyage reliées aux maladies touchant les voyageurs canadiens et les migrants récemment revenus du Brésil, qui se sont présentés dans un établissement d'un réseau de cliniques de santé voyage au Canada.

Méthodologie : Analyse des données concernant les voyageurs canadiens et les migrants de retour au pays qui se sont présentés dans un établissement du réseau CanTravNet pour recevoir des soins en raison d'une maladie entre juin 2013 et juin 2016.

Résultats : Au cours de la période de l'étude, 7707 voyageurs et migrants malades se sont présentés dans un établissement du réseau CanTravNet et $89(0,01 \%)$ avaient contracté la maladie au Brésil. Les touristes étaient le type de voyageurs le plus représenté $(n=45,50,6 \%$ ), suivis des personnes qui voyageaient pour " rendre visite à des amis et à des parents " $(n=14$, $15,7 \%$ ). L'âge médian était de 37 ans (intervalle < 1 à 78 ans); parmi les voyageurs, 49 étaient des hommes $(55,1 \%$ ) et 40 , des femmes (44,9\%). Parmi les 40 femmes, 26 (65\%) étaient en âge de procréer. Parmi les voyageurs, $9 \%(n=8)$ ont reçu un diagnostic d'infection à arbovirus, notamment la dengue $(n=6)$, le chikungunya $(n=1)$ et l'infection par le virus Zika $(n=1)$, alors que $14,6 \%(n=13)$ se sont présentés pour recevoir des soins en raison d'un syndrome viral non spécifique $(n=7)$, d'une maladie fébrile non spécifique $(n=1)$, d'une neuropathie périphérique $(n=1)$ et d'une éruption cutanée non spécifique $(n=4)$, soit quatre syndromes indiquant possiblement une infection par le virus Zika. Les voyageurs revenus malades du Brésil étaient plus susceptibles de se présenter pour recevoir des soins en raison d'une maladie apparentée à une infection à arbovirus ou à une infection par le virus Zika que les autres voyageurs revenus malades d'un autre pays d'Amérique du Sud (23,6\% des voyageurs par rapport à 10,5\% des voyageurs, respectivement $[p=0,0024])$.

Interprétation : L'application d'une approche épidémiologique pour étudier les maladies chez les voyageurs canadiens revenus du Brésil peut éclairer les professionnels de la santé canadiens qui reçoivent en consultation d'éventuels voyageurs ou des voyageurs revenant des Jeux olympiques. L'analyse a montré que les maladies à transmission vectorielle, comme la dengue, sont fréquentes et que, même dans ce petit groupe de voyageurs, les virus chikungunya et Zika étaient représentés. II est extrêmement important d'informer les voyageurs des mesures de prévention des piqûres de moustique avant qu'ils se rendent au Brésil.

Citation proposée : Boggild AK, Geduld J, Libman M, Yansouni CP, McCarthy AE, Hajek J, et al. Maladie chez les voyageurs canadiens et les migrants revenus du Brésil : Données de surveillance de CanTravNet, 2013 à 2016. Relevé des maladies transmissibles au Canada 2016;42:171-6. https://doi.org/10.14745/ccdr.v42i08a01f

\section{Affiliations}

1 Unité des maladies tropicales, Division des maladies infectieuses, Département de médecine, Réseau universitaire de santé et Université de Toronto, Toronto (Ontario)

2 Laboratoires de Santé publique Ontario, Santé publique Ontario Toronto (Ontario)

${ }^{3}$ Bureau des services de santé des voyageurs et aux frontières, Agence de la santé publique du Canada, Ottawa (Ontario)

${ }^{4}$ Centre de médecine tropicale J.D. Maclean, Université McGill, Montréal (Québec)

${ }^{5}$ Clinique médicale des voyageurs internationaux, Division des maladies infectieuses, Hôpital d'Ottawa et Université d'Ottawa, Ottawa (Ontario)

${ }^{6}$ Division des maladies infectieuses, Hôpital général de Vancouver, Université de la Colombie-Britannique, Vancouver (Colombie-Britannique)

${ }^{7}$ Maladies infectieuses, Vancouver Island Health Authority, Département de médecine, Université de la Colombie-Britannique, Victoria (Colombie-Britannique)

${ }^{8}$ Hôpital Saint-Luc du CHUM et Université de Montréal, Montréal (Québec)

${ }^{9}$ Section des maladies infectieuses pédiatriques, Département de pédiatrie et de médecine, Alberta Children's Hospital et Université de Calgary, Calgary (Alberta)

${ }^{10}$ Services de santé-voyage et médecine tropicale, Programme de régulation démographique et de santé publique, Office régional de la santé de Winnipeg, Winnipeg (Manitoba)

${ }^{11}$ Center for Geographic Medicine, Département de médecine, Université de l'Alabama, Birmingham (Alabama)

${ }^{12}$ Sandra A. Rotman Centre for Global Health, Toronto (Ontario)

*Correspondance : andrea.boggild@ utoronto.ca

\section{Introduction}

Au cours des trois dernières années, les virus Zika et chikungunya ont fait leur apparition dans les Amériques (1-3), et les taux de transmission de la dengue sont élevés partout dans les
Caraïbes, l'Amérique centrale et l'Amérique du Sud (4). Le Brésil, en particulier, a souffert de graves conséquences sanitaires et économiques résultant de l'émergence de ces virus tant en milieu urbain qu'en milieu rural (5). Les taux élevés de détection 
du virus de Zika dans l'État de Pernambuco au Brésil ont amené à prendre conscience de l'existence d'un nouveau syndrome neurologique congénital ayant des effets dévastateurs (6).

Compte tenu de la crise actuelle associée au virus Zika, on a accordé une grande attention aux Jeux olympiques de 2016 qui doivent commencer en août, à Rio de Janeiro. Le débat entourant les risques auxquels sont exposés les athlètes, leur entourage et les Canadiens, qui découlent des maladies infectieuses touchant les voyageurs revenant au Canada, a été houleux $(7,8)$. On ne dispose pas de suffisamment de renseignements sur les problèmes de santé auxquels peuvent être confrontés les Canadiens en voyage au Brésil. Afin de corriger cette lacune en matière de connaissances, les auteurs ont produit un rapport sommaire de surveillance des maladies touchant les voyageurs canadiens et les migrants revenus du Brésil, qui se sont présentés dans les établissements du réseau CanTravNet au cours d'une période de trois ans afin de recevoir des soins.

\section{Méthodologie}

Source des données : Sept établissements canadiens offrant des soins de santé après le voyage situés dans de grands centres urbains de cinq provinces (Colombie-Britannique, Alberta, Manitoba, Ontario et Québec) font partie d'un réseau mondial de surveillance appelé GeoSentinel et constituent un groupe de surveillance nationale appelé CanTravNet (9). Les données démographiques et de voyage ont été recueillies au moyen de la plateforme de collecte de données du réseau de surveillance GeoSentinel (10). Le protocole de collecte de données de GeoSentinel fait l'objet d'un examen périodique par l'agent du comité d'examen éthique du National Center for Emerging and Zoonotic Infectious Diseases des Centers for Disease Control and Prevention (CDC) des États-Unis. II est classé en tant que protocole de surveillance de la santé publique et non comme protocole de recherche sur des sujets humains et doit être soumis au comité d'examen éthique aux fins d'approbation. Les diagnostics définitifs comprennent des étiologies ( $p$. ex. virus Zika) et des syndromes précis ( $p$. ex. éruption cutanée). Tous les établissements du réseau CanTravNet ont fourni, dans la mesure du possible, des données microbiologiques confirmées, d'après les plus fiables diagnostics de référence accessibles.

Définitions et classifications : Sept motifs de voyage ont été utilisés : tourisme, affaires, missionnaire/bénévole/chercheur/ travailleur humanitaire, visite à des amis et à des parents, migration, éducation et soins médicaux prévus (9-11).

Critères d'inclusion : Les données démographiques, cliniques et de voyage provenant des Canadiens qui, à leur retour du Brésil, se sont présentés pour une consultation médicale à l'un des six établissements du réseau CanTravNet entre le 1er juin 2013 et le $1^{\text {er }}$ juin 2016 ont été extraites et analysées. Seuls les patients ayant reçu un diagnostic définitif probable ou confirmé ont été inclus.
Analyse : Les données extraites ont été gérées par une base de données Microsoft Access. Les voyageurs ont été classés par motif de voyage, caractéristique démographique et de voyage, y compris la consultation avant le voyage et le diagnostic. Les femmes en âge de procréer ont été définies comme étant celles âgées de 15 à 49 ans. Les différences entre les groupes de voyageurs ont été comparées au moyen du test exact de Fisher. On a effectué tous les calculs statistiques à l'aide du logiciel GraphPad Prism (GraphPad Software Inc., La Jolla, Californie).

\section{Résultats}

Au cours de la période de l'étude, 7707 voyageurs et migrants malades se sont présentés dans un établissement du réseau CanTravNet et $89(0,01 \%)$ avaient contracté la maladie au Brésil. Les touristes étaient le type de voyageurs le plus représenté ( $n=45,50,6 \%$ ), suivis des personnes ayant voyagé pour rendre visite à des amis et à des parents $(n=14,15,7 \%)$ et pour affaires ( $n=13,14,6 \%)$, des migrants ( $n=6,6,7 \%)$, des missionnaires, bénévoles, chercheurs ou travailleurs humanitaires $(n=6,6,7 \%)$, des personnes ayant voyagé à des fins d'éducation $(n=3,3,4 \%)$ ou pour recevoir des soins médicaux prévus ( $n=2,2,2 \%$ ).

L'âge médian était de 37 ans (intervalle $<1$ à 78 ans); parmi les voyageurs, 49 étaient des hommes $(55,1 \%)$ et 40 , des femmes $(44,9 \%)$. Six voyageurs $(6,7 \%)$ avaient moins de 18 ans. Les principaux pays de naissance étaient le Canada ( $n=50,56,2 \%$ ) et le Brésil ( $n=21,23,6 \%$ ). La durée médiane du voyage était de 16 jours (intervalle de 3 à 304 jours). Près d'un tiers des voyageurs ( $n=28,31,5 \%)$ ont été vus en consultation avant le voyage.

Presque $98 \%$ des voyageurs et des migrants revenus malades au pays qui ont été inclus dans cette analyse ont été traités en consultation externe $(n=87)$. Les symptômes les plus courants étaient les symptômes dermatologiques ( $n=43,48,3 \%$ ), suivis des symptômes gastro-intestinaux ( $n=37,41,6 \%$ ) et de la fièvre ( $n=21,23,6 \%)$. La larva migrans cutanée $(n=8,9,0 \%)$, les piqûres d'arthropodes $(n=8,9,0 \%$ ) et le syndrome du côlon irritable post-infectieux ( $n=10,11,2 \%$ ) étaient les diagnostics dermatologiques et gastro-intestinaux les plus courants, respectivement (tableau 1). Des maladies à transmission féco-orale selon toute vraisemblance, comme la fièvre typhoïde, la diarrhée aiguë, l'amibiase, la giardiase, la campylobactériose et la shigellose, ont touché $10 \%(n=9)$ des voyageurs revenus malades au pays. Des maladies respiratoires, comme la grippe, le syndrome pseudogrippal, la pneumonie lobaire et les infections des voies respiratoires supérieures, sont survenues chez 7,9\% $(n=7)$ des voyageurs revenus malades au pays.

Des affections fébriles, dont des infections à arbovirus, sont survenus chez $9 \%(n=8)$ des voyageurs ayant reçu un diagnostic de dengue $(n=6)$, de chikungunya $(n=1)$ ou d'infection par le virus Zika $(n=1)$. Les huit voyageurs $(100 \%)$ atteints du chikunguyna, de la dengue ou d'une infection par le virus Zika avaient emprunté un itinéraire passant par le Brésil pendant les mois de janvier à juin, alors que seulement deux de ces huit voyageurs (25\%) s'étaient rendus au Brésil pendant les mois de 
Tableau 1 : Caractéristiques démographiques des 89 voyageurs et migrants revenus au pays, qui se sont présentés dans un établissement du réseau CanTravNet pour recevoir des soins en raison d'une maladie contractée au Brésil, 2013 à 2016

\begin{tabular}{|c|c|c|c|c|c|c|c|c|}
\hline \multirow[t]{2}{*}{ Diagnostic } & \multicolumn{2}{|c|}{$\begin{array}{l}\text { Tous les voyageurs } \\
\qquad n=89\end{array}$} & \multicolumn{2}{|c|}{$\begin{array}{c}\text { Voyageurs } \\
\text { faisant } \\
\text { un court } \\
\text { séjour } \\
\text { (voyage } \leq 2 \\
\text { semaines) } \\
n=26\end{array}$} & \multicolumn{2}{|c|}{$\begin{array}{l}\text { Voyageurs } \\
\text { faisant un } \\
\text { séjour de } \\
\text { moyenne } \\
\text { durée }^{2} \\
\text { (voyage }^{\text {(voge }} \\
2-4 \text { semaines) } \\
n=24\end{array}$} & \multicolumn{2}{|c|}{$\begin{array}{c}\text { Voyageurs } \\
\text { faisant } \\
\text { un séjour } \\
\text { prolongé } \\
\text { (voyage } \\
\geq 1 \text { mois) } \\
n=24\end{array}$} \\
\hline & $n$ & $\%$ & $n$ & $\%$ & $n$ & $\%$ & $\mathrm{n}$ & $\%$ \\
\hline \multicolumn{9}{|c|}{ Maladie systémique fébrile } \\
\hline $\begin{array}{l}\text { Syndrome viral } \\
\text { non spécifique } \\
\text { ou syndrome } \\
\text { apparenté à la } \\
\text { mononucléose }\end{array}$ & 7 & 7,9 & 2 & 7,7 & 4 & 16,7 & 1 & 4,2 \\
\hline Dengue & 6 & 6,7 & 0 & 0 & 3 & 12,5 & 3 & 12,5 \\
\hline $\begin{array}{l}\text { Grippe et } \\
\text { syndrome } \\
\text { pseudogrippal }\end{array}$ & 4 & 4,5 & 3 & 11,5 & 0 & 0 & 0 & 0 \\
\hline $\begin{array}{l}\text { Infection des } \\
\text { voies respiratoires } \\
\text { supérieures }\end{array}$ & 2 & 2,2 & 0 & 0 & 0 & 0 & 2 & 8,3 \\
\hline $\begin{array}{l}\text { Fièvre entérique } \\
\text { due à } \\
\text { Salmonella typhi }\end{array}$ & 1 & 1,1 & 0 & 0 & 1 & 4,2 & 0 & 0 \\
\hline Pneumonie lobaire & 1 & 1,1 & 1 & 3,8 & 0 & 0 & 0 & 0 \\
\hline Fièvre chikungunya & 1 & 1,1 & 1 & 3,8 & 0 & 0 & 0 & 0 \\
\hline $\begin{array}{l}\text { Infection par le } \\
\text { virus Zika }\end{array}$ & 1 & 1,1 & 0 & 0 & 0 & 0 & 1 & 4,2 \\
\hline $\begin{array}{l}\text { Syndrome } \\
\text { apparenté à } \\
\text { l'infection par le } \\
\text { virus Zika }\end{array}$ & 13 & 14,6 & 2 & 7,7 & 6 & 25,0 & 3 & 12,5 \\
\hline Méningite virale & 1 & 1,1 & 0 & 0 & 1 & 4,2 & 0 & 0 \\
\hline \multicolumn{9}{|c|}{ Maladie gastro-intestinale } \\
\hline $\begin{array}{l}\text { Syndrome du côlon } \\
\text { irritable } \\
\text { post-infectieux }\end{array}$ & 10 & 11,2 & 1 & 3,8 & 3 & 12,5 & 3 & 12,5 \\
\hline Strongyloidiose & 4 & 4,5 & 0 & 0 & 0 & 0 & 2 & 8,3 \\
\hline Diarrhée aiguë & 3 & 3,4 & 2 & 7,7 & 0 & 0 & 1 & 4,2 \\
\hline Diarrhée chronique & 2 & 2,2 & 0 & 0 & 0 & 0 & 1 & 4,2 \\
\hline $\begin{array}{l}\text { Amibiase causée } \\
\text { par Entamoeba } \\
\text { histolytica }\end{array}$ & 2 & 2,2 & 1 & 3,8 & 0 & 0 & 1 & 4,2 \\
\hline Giardiase & 1 & 1,1 & 0 & 0 & 0 & 0 & 0 & 0 \\
\hline Shigellose & 1 & 1,1 & 1 & 3,8 & 0 & 0 & 0 & 0 \\
\hline Campylobactériose & 1 & 1,1 & 1 & 3,8 & 0 & 0 & 0 & 0 \\
\hline \multicolumn{9}{|c|}{ Maladie dermatologique } \\
\hline $\begin{array}{l}\text { Larva migrans } \\
\text { cutanée }\end{array}$ & 8 & 9,0 & 2 & 7,7 & 4 & 16,7 & 2 & 8,3 \\
\hline Piqûre d'arthropode & 8 & 9,0 & 4 & 15,4 & 3 & 12,5 & 1 & 4,2 \\
\hline $\begin{array}{l}\text { Infection de la peau } \\
\text { et des tissus mous }{ }^{4}\end{array}$ & 6 & 6,7 & 2 & 7,7 & 1 & 4,2 & 2 & 8,3 \\
\hline $\begin{array}{l}\text { Éruption cutanée } \\
\text { d'étiologie inconnue }\end{array}$ & 4 & 4,5 & 0 & 0 & 2 & 8,3 & 1 & 4,2 \\
\hline Morsure d'animal $^{5}$ & 2 & 2,2 & 1 & 3,8 & 0 & 0 & 1 & 4,2 \\
\hline $\begin{array}{l}\text { Leishmaniose } \\
\text { cutanée }\end{array}$ & 2 & 2,2 & 0 & 0 & 0 & 0 & 1 & 4,2 \\
\hline
\end{tabular}

Abréviation : n, nombre

La cohorte totale de voyageurs comprenait 7707 personnes ayant voyagé entre le 1er juin 2013 et le $1^{\text {er }}$ juin 2016

${ }^{2}$ Comprend les personnes ayant contracté une maladie lors d'un voyage au Brésil au cours de l'année précédant la présentation $(n=74)$. Exclut les migrants $(n=6)$ et les personnes ayant contracté une maladie lors d'un voyage au Brésil plus d'un an avant la présentation $(n=9)$

${ }^{3}$ Inclut le syndrome viral, l'éruption cutanée non spécifique, le syndrome fébrile non spécifique et la neuropathie périphérique. Exclut les cas confirmés d'infection par le virus Zika

${ }^{4}$ Inclut l'impétigo, l'ecthyma, la paronychie, la folliculite, l'érysipèle, la furonculose, la carbunculose et la cellulite

${ }^{5}$ Inclut les morsures de singes et de chiens
Figure 1 : Huit cas d'infection à arbovirus contractés au Brésil par mois de voyage, CanTravNet, 2013 à 2016

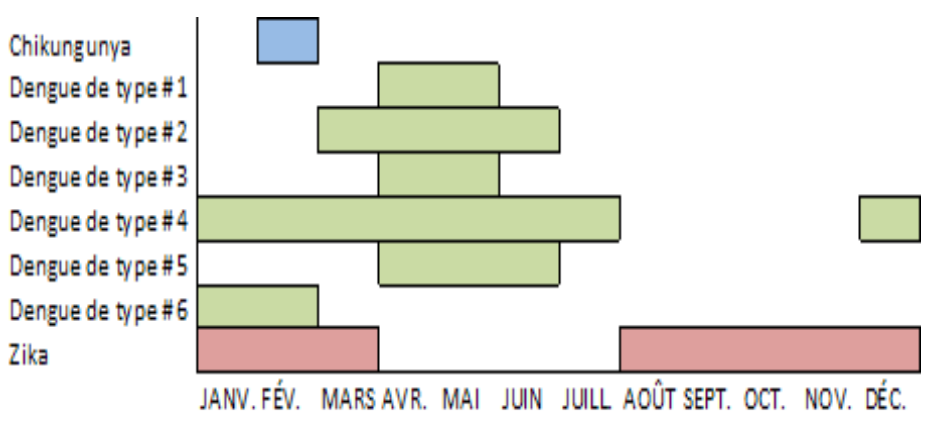

Remarque : La plupart des cas d'infection à arbovirus ont été contractés entre décembre et juin plutôt qu'en juin et septembre, moment de la tenue des Jeux olympiques de 2016

juillet à décembre (figure 1). Le syndrome viral non spécifique $(n=7)$, l'éruption cutanée non spécifique $(n=4)$, le syndrome fébrile non spécifique $(n=1)$ et la neuropathie périphérique $(n=1)$, quatre syndromes indiquant possiblement une infection par le virus Zika, étaient également bien représentés (tableau 1).

Parmi les 40 femmes ayant voyagé au Brésil, 26 (65\%) étaient en âge de procréer. À peine plus du tiers des femmes en âge de procréer ont été vues en consultation avant le voyage $(n=9,34,6 \%)$. Les taux d'infection à arbovirus chez les femmes en âge de procréer semblaient comparables à ceux notés chez les autres voyageurs : $2 / 26(7,7 \%)$ par rapport à $6 / 63(9,5 \%)$, respectivement $(p=1,00)$. Les taux d'éruption cutanée non spécifique semblaient toutefois plus élevés chez les femmes en âge de procréer que chez les autres voyageurs : $3 / 26$ (11,5\%) par rapport à 1/63 $(1,6 \%)$, respectivement, bien que cette différence $n$ 'ait pas été significative $(p=0,07)$. Les femmes en âge de procréer semblaient plus enclines à recevoir un diagnostic possiblement compatible avec une maladie apparentée à une infection par le virus Zika, y compris une neuropathie périphérique, un syndrome viral, un syndrome fébrile non spécifique et une éruption cutanée, que les autres voyageurs : $6 / 26(23,1 \%)$ par rapport à $7 / 63(11,1 \%)$, respectivement, bien que cette différence n'ait pas été significative $(p=0,189)$.

Comparativement aux autres voyageurs et migrants revenus malades d'un autre pays d'Amérique du Sud $(n=401)$, ceux ayant contracté une maladie au Brésil étaient plus enclins à recevoir un diagnostic de dengue, de chikungunya ou d'infection par le virus Zika : 8/89 (9,0\%) par rapport à 13/401 (3,2\%) $(p=0,0362)$ (tableau 2). De plus, les voyageurs revenus malades du Brésil étaient plus susceptibles que les autres voyageurs revenus malades d'un autre pays d'Amérique du Sud d'avoir contracté une maladie apparentée à une infection par le virus Zika : 13/89 (14,6 \%) par rapport à 29/401 (7,2\%) $(p=0,0347)$ (tableau 2). 
Tableau 2 : Infections à arbovirus et maladies apparentées à une infection à arbovirus chez les voyageurs et les migrants revenus malades du Brésil ( $n=89$ ) et d'un autre pays d'Amérique du Sud $(n=401)$, qui se sont présentés dans un établissement du réseau CanTravNet, 2013 à 2016

\begin{tabular}{|c|c|c|c|c|c|}
\hline \multirow[t]{2}{*}{$\begin{array}{l}\text { Diagnostic } \\
\text { ou groupe de } \\
\text { diagnostics }\end{array}$} & \multicolumn{2}{|c|}{$\begin{array}{c}\text { Voyageurs et } \\
\text { migrants revenus } \\
\text { malades du Brésil } \\
(n=89)\end{array}$} & \multicolumn{2}{|c|}{$\begin{array}{l}\text { Voyageurs et migrants } \\
\text { revenus malades d'un } \\
\text { autre pays d'Amérique } \\
\text { du Sud ( } n=401 \text { ) }\end{array}$} & \multirow[t]{2}{*}{ Valeur $p$} \\
\hline & $n$ & $\begin{array}{c}\text { Nombre par } \\
100 \text { voyageurs }\end{array}$ & $n$ & $\begin{array}{l}\text { Nombre par } \\
100 \text { voyageurs }\end{array}$ & \\
\hline $\begin{array}{l}\text { A. Infection } \\
\text { à arbovirus } \\
\text { (dengue, CHK ou } \\
\text { infection par le } \\
\text { virus Zika) }^{1}\end{array}$ & 8 & 9 & 13 & 3,2 & $p=0,0362$ \\
\hline $\begin{array}{l}\text { B. Maladie } \\
\text { apparentée à une } \\
\text { infection par le } \\
\text { virus Zika² }\end{array}$ & 13 & 14,6 & 29 & 7,2 & $p=0,0347$ \\
\hline Total A + B & 21 & 23,6 & 42 & 10,5 & $p=0,0024$ \\
\hline
\end{tabular}

Abréviation : $\mathrm{n}$, nombre; $\mathrm{CHK}$, chikungunya

Diagnostic confirmé en laboratoire

2 Diagnostic clinique incluant un syndrome viral non spécifique, une maladie fébrile non

spécifique, une éruption cutanée non spécifique et une neuropathie périphérique

\section{Discussion}

Cette analyse des données de surveillance aide à comprendre les détails démographiques et de voyage d'un sous-groupe de voyageurs canadiens et de migrants revenus malades au pays après avoir contracté une maladie au Brésil et peut éclairer les professionnels de la santé canadiens qui reçoivent en consultation d'éventuels voyageurs ou des voyageurs revenant des Jeux olympiques de 2016. Ces données mettent en évidence la récente émergence des infections à arbovirus, comme la dengue, le chikungunya et l'infection par le virus Zika, à laquelle on assiste actuellement chez les voyageurs en Amérique centrale et en Amérique du Sud ainsi que le taux élevé de piqûres d'arthropodes chez les voyageurs au Brésil.

Parmi les voyageurs revenus malades du Brésil, $9 \%$ étaient atteints d'une maladie virale à transmission vectorielle et $10 \%$ avaient subi une piqûre d'arthropode

Les maladies à transmission vectorielle, dont la dengue, le chikungunya et l'infection par le virus Zika, étaient bien représentées dans ce groupe de voyageurs revenus malades du Brésil, et semblaient avoir été associées principalement à des voyages pendant les mois de janvier à juin plutôt que pendant la dernière moitié de l'année, période pendant laquelle les Jeux olympiques seront tenus. Comparativement aux voyageurs et aux migrants revenus malades d'un autre pays d'Amérique du Sud, les personnes ayant voyagé au Brésil étaient deux à trois fois plus susceptibles de contracter une maladie apparentée à une infection à arbovirus ou à une infection par le virus Zika. En raison du manque actuel de disponibilité du vaccin ou de chimioprophylaxie, la prévention des infections à arbovirus, comme la dengue, le chikungunya et l'infection à virus Zika, repose sur l'application de mesures de prévention des piqûres de moustique comme un hébergement muni de moustiquaires, des vêtements imprégnés d'insectifuge et des insectifuges contenant du DEET ou de l'icaridine $(12,13)$. La transmission sexuelle du virus Zika peut être limitée par I'utilisation du condom et l'abstinence $(6,14)$.

\section{Près de $15 \%$ des voyageurs revenus malades du Brésil étaient atteints d'une maladie apparentée à une infection par le virus Zika}

Bien que des études phylogéniques aient permis d'établir clairement que l'arrivée du virus Zika au Brésil a eu lieu en 2013 (15), le dépistage du virus Zika n'est possible que depuis peu de temps (14). Un total de $15 \%$ de voyageurs revenus malades du Brésil, ayant été inclus dans cette analyse, ont reçu un diagnostic indiquant possiblement la présence du virus Zika, notamment un syndrome viral ou fébrile non spécifique, une éruption cutanée non spécifique et une neuropathie périphérique. Les voyageurs qui se sont présentés dans un établissement du réseau CanTravNet tôt au début de la période de l'étude avant que la transmission de Zika dans les Amériques soit reconnue n'ont pas fait l'objet d'un test de dépistage du virus Zika. De plus, compte tenu du long délai de dépistage du virus Zika (14), de nombreux voyageurs ayant récemment reçu un diagnostic de maladie apparentée à une infection par le virus Zika pourraient, dans le futur, recevoir un résultat positif au test de confirmation. Un fait encore plus préoccupant est la découverte d'un syndrome apparenté à l'infection par le virus Zika chez presque un quart des femmes en âge de procréer. Compte tenu de la fréquence et de la gravité du syndrome congénital nouvellement reconnu attribuable au virus Zika $(6,14)$, il est conseillé aux femmes enceintes d'éviter de se rendre aux Jeux olympiques $(14,16)$ et à celles qui souhaitent le devenir d'envisager de reporter leur voyage (14).

\section{Limites}

L'analyse des données du réseau CanTravNet a plusieurs limites (9). Cette analyse ne se rapporte qu'à l'échantillon de voyageurs revenus malades au pays qui se sont présentés dans un établissement du réseau CanTravNet à la suite d'un voyage au Brésil. Par conséquent, il est possible que nos conclusions ne puissent être généralisées à d'autres voyageurs canadiens et aux personnes se rendant dans d'autres pays. La surreprésentation apparente des maladies apparentées à une infection à arbovirus ou à une infection par le virus Zika chez les femmes en âge de procréer peut simplement être liée au biais lié au processus d'aiguillage à la suite de l'émergence du virus Zika dans les Amériques. Notre base de données peut sous-représenter les voyageurs ayant contracté une maladie de courte durée pendant un séjour prolongé, puisqu'il est possible que ces personnes aient passé leur convalescence à l'étranger et n'aient pas demandé à recevoir des soins à leur retour au pays. Nos données ne permettent pas d'estimer le taux d'incidence d'un diagnostic précis ou de chiffrer précisément les risques de contracter ladite maladie à une destination (17). Puisque l'établissement de Winnipeg a été intégré au réseau CanTravNet en 2016, les voyageurs du Manitoba revenus au pays ne sont pas représentés. Dans le cas de 31,5\% voyageurs malades de retour au pays, on ne dispose pas de données sur une consultation de santé avant le voyage. Finalement, compte tenu de la nature de notre réseau, les cas pédiatriques sont sous-représentés. Par conséquent, nos données pourraient ne pas s'appliquer à la population pédiatrique canadienne qui voyage. 


\section{Conclusion}

Les données de surveillance de CanTravNet peuvent être utilisées pour mieux éclairer les professionnels de la santé qui conseillent les éventuels voyageurs se rendant au Brésil ainsi que l'approche diagnostique chez les voyageurs et les migrants malades de retour au pays après un séjour au Brésil. Ces données mettent en évidence l'émergence des virus chikungunya et Zika chez les voyageurs au Brésil et le fait que la dengue demeure une infection à arbovirus fréquente chez les voyageurs en Amérique du Sud. La fréquence des infections à arbovirus et des piqûres d'arthropodes chez ce groupe de voyageurs au Brésil attire l'attention sur la nécessité de prendre de grandes précautions pour prévenir les piqûres d'insectes surtout au cours de la journée. Le renforcement du nombre et du type de mesures de prévention des piqûres de moustique offertes aux personnes qui désirent se rendre au Brésil devrait être l'un des principaux points à aborder lors des discussions précédant un voyage.

\section{Contributions}

AKB a conçu l'étude, a participé à l'élaboration de l'étude ainsi qu'à la collecte, à l'analyse et à l'interprétation des données et a été le rédacteur principal de l'article. JG a participé à la conception de l'étude, à l'interprétation des données ainsi qu'à I'évaluation critique et à la révision de l'article. JV a participé à la collecte, à l'analyse et à l'interprétation des données ainsi qu'à I'évaluation critique et à la révision de l'article. $M L, C Y, A E M, J H$, WG, SK et KCK ont participé à la collecte et à l'interprétation des données ainsi qu'à l'évaluation critique et à la révision de I'article. DOF et PJP ont participé à l'interprétation des données ainsi qu'à l'évaluation critique et à la révision de l'article. Tous les auteurs assument la responsabilité de l'intégrité de l'article.

\section{Conflit d'intérêts}

Aucun.

\section{Financement}

CanTravNet est le réseau correspondant de médecine tropicale et de médecine de voyage de l'Agence de la santé publique du Canada, qui a été financé par le Bureau des services de santé des voyageurs et aux frontières, une division de I'ASPC. Il a été créé par la fusion des centres canadiens de GeoSentinel, le réseau mondial de surveillance de l'International Society of Travel Medicine (ISTM) appuyé par l'accord de coopération U50/CCU412347 conclu entre les CDC et I'ISTM. La source de financement de GeoSentinel n'a joué aucun rôle dans l'élaboration de l'étude, l'analyse et l'interprétation des données et la rédaction de l'article. La source de financement de CanTravNet a, quant à elle, contribué à l'élaboration de l'étude et à l'évaluation critique de l'article, mais n'a pas eu accès aux données brutes.

\section{Références}

1. Deilgat M, Geduld J, Drebot, M. Éclosion de chikungunya dans les Caraïbes (2013-2014). Relevé des maladies transmissibles au Canada 2014;40:7-12. http://www.phacaspc.gc.ca/publicat/ccdr-rmtc/14vol40/dr-rm40-02/dr-rm4002-chik-fra.php.

2. Drebot MA, Holloway K, Zheng H, Ogden NH. Cas de chikungunya liés aux voyages au Canada, 2014. Relevé des maladies transmissibles au Canada 2015;41:2-6. http://www. phac-aspc.gc.ca/publicat/ccdr-rmtc/15vol41/dr-rm41-01/rapidfra.php.

3. Agence de la santé publique du Canada. Infection à virus Zika: Situation mondiale - Conseils de santé aux voyageurs. Le 27 mai 2016. Ottawa : ASPC; 2016 [Date de modification: 27 avril 2016]. https://travel.gc.ca/travelling/health-safety/ travel-health-notices/152.

4. Rodriguez-Morales AJ, Villamil-Gómez WE, Franco-Paredes C. The arboviral burden of disease caused by co-circulation and co-infection of dengue, chikungunya and Zika in the Americas. Travel Med Infect Dis 2016;14(3):177-9.

5. Constenla D, de Broucker G, del Campo JM. The potential economic impact of the Zika virus. Dengue Vaccine Initiative (DVI) - Winter newsletter 2016. Washington: DVI; 2016 [updated 2016 April 27; cited 2016 Apr 19]. http://www. denguevaccines.org/winter-newsletter-2016\#/zika.

6. Panchaud A, Stojanov M, Ammerdorffer A, Vouga M, Baud D. Emerging role of Zika virus in adverse fetal and neonatal outcomes. Clin Microbiol Rev 2016 Jul;29(3):659-94.

7. Petersen E, Wilson ME, Touch S, McCloskey B, Mwaba P, Bates $M$, et.al. Rapid spread of Zika virus in the Americas: Implications for public health preparedness for mass gatherings at the 2016 Brazil Olympic Games. Int J Infect Dis 2016 Mar;44:11-5. doi: 10.1016/j.ijid.2016.02.001.

8. Coombes R. Call to cancel 2016 Olympics because of Zika risk is not backed by WHO guidance. BMJ 2016 May 20;353:i2899. doi: 10.1136/bmj.i2899.

9. Boggild AK, Geduld J, Libman M, McCarthy A, Vincelette J, Ghesquiere W, et.al. Infections contractées en voyage au Canada : réseau CanTravNet 2011-2012. Relevé des maladies transmissibles au Canada 2014;40(16):313-27. http://www.phac-aspc.gc.ca/publicat/ccdr-rmtc/14vol40/drrm40-16/dr-rm40-16-surv-fra.php.

10. Leder K, Torresi J, Libman M, Cramer JP, Castelli F, Schlagenhauf $P$ et al. GeoSentinel surveillance of illness in returned travelers, 2007-2011. Ann Int Med 2013;158:45668.

11. Leder K, Tong S, Weld L, Kain KC, Wilder-Smith A, von Sonnenburg $F$, et al. Illness in travelers visiting friends and relatives: A review of the GeoSentinel Surveillance Network. Clin Infect Dis 2006;43:1185-93.

12. Schofield S, Plourde $P$, au nom du Comité consultatif de la médecine tropicale et de la médecine des voyages. Déclaration relative aux mesures de protection individuelle pour prévenir les piqûres ou morsures d'arthropodes. Relevé des maladies transmissibles au Canada 2012;38(DCC-3):120. http://www.phac-aspc.gc.ca/publicat/ccdr-rmtc/12vol38/ acs-dcc-3/index-fra.php. 
13. Rodriguez SD, Drake LL, Price DP, Hammond JI, Hansen IA. The efficacy of some commercially available insect repellents for Aedes aegypti (Diptera: Culicidae) and Aedes albopictus (Diptera: Culicidae). J Insect Sci 2015 Oct;15(1):140. doi: 10.1093/jisesa/iev125.

14. Groupe de travail sur Zika du Comité consultatif de la médecine tropicale et de la médecine des voyages (CCMTMV). Recommandations canadiennes pour la prévention et le traitement du virus Zika : mise à jour: 14 juin 2016. Relevé des maladies transmissibles au Canada 2016;42:114-25. http://www.phac-aspc.gc.ca/publicat/ccdrrmtc/16vol42/dr-rm42-5/ar-01-fra.php.
15. Faria NR, Azevedo Rdo S, Kraemer MU, Souza R, Cunha MS, Hill SC, et.al. Zika virus in the Americas: Early epidemiological and genetic findings. Science $2016 \mathrm{Apr}$ 15;352(6283):345-9. doi: 10.1126/science.aaf5036.

16. Agence de la santé publique du Canada. Jeux olympiques et paralympiques d'été de 2016 à Rio de Janeiro, au Brésil Conseils de santé au voyageurs. Mise à jour : le 14 juin 2016. Ottawa: ASPC; 2016. http://www.phac-aspc.gc.ca/tmp-pmv/ notices-avis/notices-avis-fra.php?id=153.

17. Leder K, Steffen R, Cramer JP, Greenaway C. Risk assessment in travel medicine: How to obtain, use, and interpret risk data for informing pre-travel advice. J Travel Med 2014; Nov 6. doi: 10.1111/jtm.12170.

\section{APPEL DE PRÉSENTATIONS}

\section{Avez-vous créé un nouveau programme intéressant dont les résultats s'avèrent prometteurs?}

Décrivez votre travail et il pourrait faire l'objet d'une publication dans notre numéro du printemps 2017 sur la science de la mise en œuvre.

\section{La date limite des soumissions est} le 10 novembre 2016.

Pour obtenir des conseils sur l'article, veuillez visiter notre page Soumettre un article en ligne :

phac-aspc.gc.ca/publicat/ccdr-rmtc/ index-fra.php

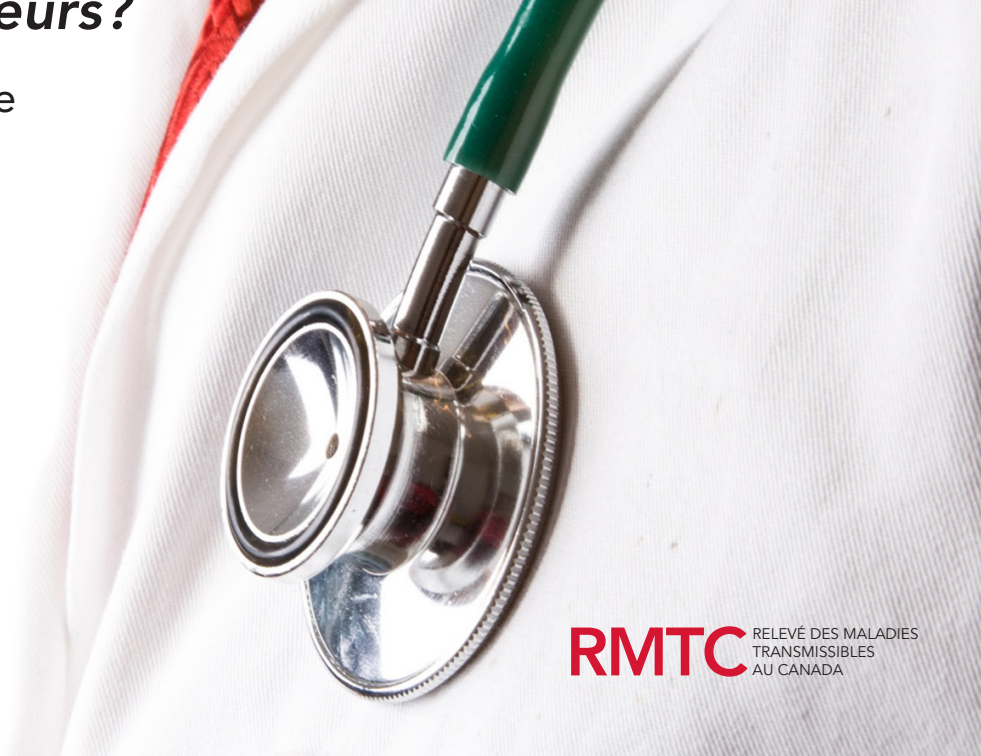

